\title{
Word level and efficient text recognition from natural scene images
}

\author{
Dhiveha.S ${ }^{1}$, Sujatha. $M^{2}$ \\ Student, Computer Science and Engineering, St.Joseph's College of Engineering and Technology, Thanjavur, India ${ }^{1}$ \\ Asst.Prof., Computer Science and Engineering, St.Joseph's College of Engineering and Technology,Thanjavur,India ${ }^{2}$
}

\begin{abstract}
Text and strings in images will be used to provide more information's. Extracting text directly from natural scene images or videos is a challenging task because of diverse text patterns and variant background interferences. Text recognition from natural images can be made using discriminative character descriptor and character structure. But there is a chance of false recognition and low text accuracy. In this paper, the accuracy rate of text detection and adding lexicon analysis is done to extend our system to word-level recognition in natural videos. To improve the accuracy and practicality of scene text extraction, designing more representative and discriminative features to model text structure will be made. This can be achieved by collecting a database of specific scene text words as stronger training set, for example, a set of word patches "EXIT" or "SALE" cropped from scene images. In addition, we will combine scene text extraction with other techniques like content-based image retrieval to develop more useful vision based assistant system.
\end{abstract}

Keywords: Scene text detection, scene text recognition, mobile application, character descriptor, text understanding, text retrieval.

\section{INTRODUCTION}

Text from most of the images are not recognized properly by which it can be used for valuable tasks. In natural scene images and videos, text characters and strings usually appear in nearby sign boards and hand-held objects and provide significant knowledge of surrounding environment and objects. To extract text information by mobile devices from natural scene, automatic and efficient scene text detection and recognition algorithms are essential. However, extracting scene text is a challenging task due to two main factors: 1) cluttered backgrounds with noise and non-text outliers, and 2) diverse text patterns such as character types, fonts, and sizes. The frequency of occurrence of text in natural scene is very low, and a limited number of text characters are embedded into complex non-text background outliers. Background textures, such as grid, window, and brick, even resemble text characters and strings. For example, a frontal face normally contains a mouth, a nose, two eyes, and two brows as prior knowledge. However, it is difficult to model the structure of text characters in scene images due to the lack of discriminative pixel-level appearance and structure features from non-text background outliers. Further, text consists of different words where each word may contain different characters in various fonts, styles, and sizes, resulting in large intra-variations of text patterns. To solve these challenging problems, scene text extraction is divided into two processes: text detection and text recognition. Pixel-based layout analysis is adopted to extract text regions and segment text characters in images, based on color uniformity and horizontal alignment of text characters. The first one is training a character recognizer to predict the category of a character in an image patch. The second one is training a binary character classifier for each character class to predict the existence of this category in an image patch. The two schemes are compatible with two promising applications related to scene text, which are text understanding and text retrieval. Text understanding is to acquire text information from natural scene to understand surrounding environment and objects. Text retrieval is to verify whether a piece of text information exists in natural scene. These two applications can be widely used in smart mobile device. The main contributions of this paper are associated with the proposed two recognition schemes. Firstly, a character descriptor is proposed to extract representative and discriminative features from character patches. It combines several feature detectors (Harris-Corner, Maximal Stable Extremal Regions (MSER), and dense sampling) and Histogram of Oriented Gradients (HOG) descriptors .Secondly, to generate a binary classifier for each character class in text retrieval, we propose a novel stroke configuration from character boundary and skeleton to model character structure.

\section{RELATED WORK}

A general review of previous work on scene text recognition is discussed here. While text detection aims to localize text regions in images by filtering out non text outliers from cluttered background text recognition is to transform image-based text information in the detected regions into readable text codes. Scene text on Document Analysis and Recognition (ICDAR) .

Gabor-based appearance model, a language model related to simultaneity frequency and letter case, similarity model, and lexicon model to perform scene character recognition. proposed a real time scene text localization and recognition method based on extremal regions Smith built 
a similarity model of scene text characters based on SIFT, and maximized posterior probability of similarity constraints by integer programming.

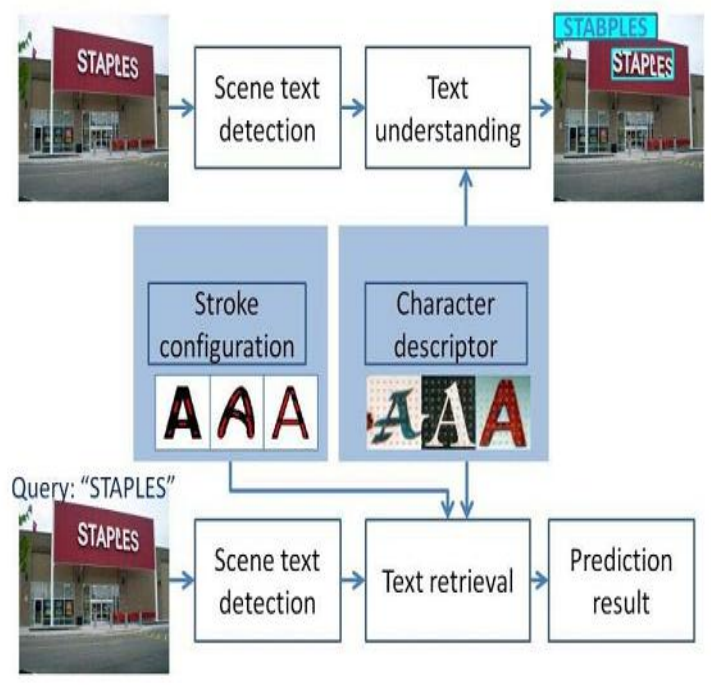

Fig. 1. The flow chart of our designed scene text extraction method

Current optical character recognition (OCR) systems can achieve almost perfect recognition rate on printed text in scanned documents, but cannot accurately recognize text information directly from camera-captured scene images and videos, and are usually sensitive to font scale changes and background interference which widely exists in scene text. Although some OCR systems have started to support scene character recognition, the recognition performance is still much lower than the recognition for scanned documents. Many algorithms were proposed to improve scene-image-based text character recognition.

In a part-based tree structure model was designed to detect text characters under Latent-SVM , and recognize text words from text regions under conditional random field. In Scale Invariant Feature Transform (SIFT) feature matching was adopted to recognize text characters in different languages, and a voting and geometric verification algorithm was presented to filter out false positive matches. In generic object recognition method was imported to extract scene text information. A dictionary of words to be spot is built to improve the accuracy of detection and recognition. Character structure was modeled by HOG features and cross correlation analysis of character similarity for text recognition and detection.

\section{III . LAYOUT-BASED TEXT DETECTION}

In natural scene, most text information is set for instruction or identifier. Text strings in print font are located at signage boards or object packages. They are normally composed of characters in uniform color and aligned arrangement, while non-text background outliers are in the form of disorganized layouts. The color To model the boundary size and location of a text string, a

uniformity and horizontal alignment were employed to bounding box is assigned to each boundary in a color localize text regions in scene images. In our current work, layer. scene text detection process is improved to be compatible with mobile applications

The boundaries of text characters are separated from those of background outliers. In each color layer, we analyze geometrical properties of the boundaries to detect the existence of text characters. According to our observation, text information generally appears in text strings composed of several character members in similar sizes rather than single character, and text strings are normally in approximately horizontal alignment. Thus we design an adjacent character grouping algorithm to search for image regions containing text strings. To model the boundary size and location of a text string, a bounding box is assigned to each boundary in a color layer. For each bounding box, we search for its siblings in similar size and vertical locations (horizontal alignment).

\section{TEXT DETECTION}

When estimating horizontal alignment, we do not require all the characters exactly align in horizontal orientation, but allow some differences between neighboring characters that are assigned into the same string. In our system we set this range as $\pm p / 6$ degrees relative to the horizontal line. This range could be set to be larger but it would bring in more false positive strings from background.

\section{A. Layout Analysis of Color Decomposition}

According to our observations, the text on sign boards or print labels on nearby objects in general appear in uniform color. Thus we can group the pixels with similar color into the same layers, and separate text from background outliers in different colors.

To decompose a scene image into several color-based layers, Text information is generally attached to a plane carrier as attachment surface with uniform colors respectively. We define the uniformity of their color difference as bigram color uniformity. Color difference is related to the character boundary, which serves as a border between text strokes and the attachment surfaces. We then model color difference by a vector of color pair, which is obtained by cascading the RGB colors of text and attachment surfaces. Each boundary can be described by a color-pair, and we cluster the boundaries with similar color pairs into the sample layer.

\section{B. Layout Analysis of Horizontal Alignment}

In each color layer, we analyze geometrical properties of the boundaries to detect the existence of text characters. According to our observation, text information generally appears in text strings composed of several character members in similar sizes rather than single character, and text strings are normally in approximately horizontal alignment. Thus we design an adjacent character grouping algorithm to search for image regions containing text strings. 


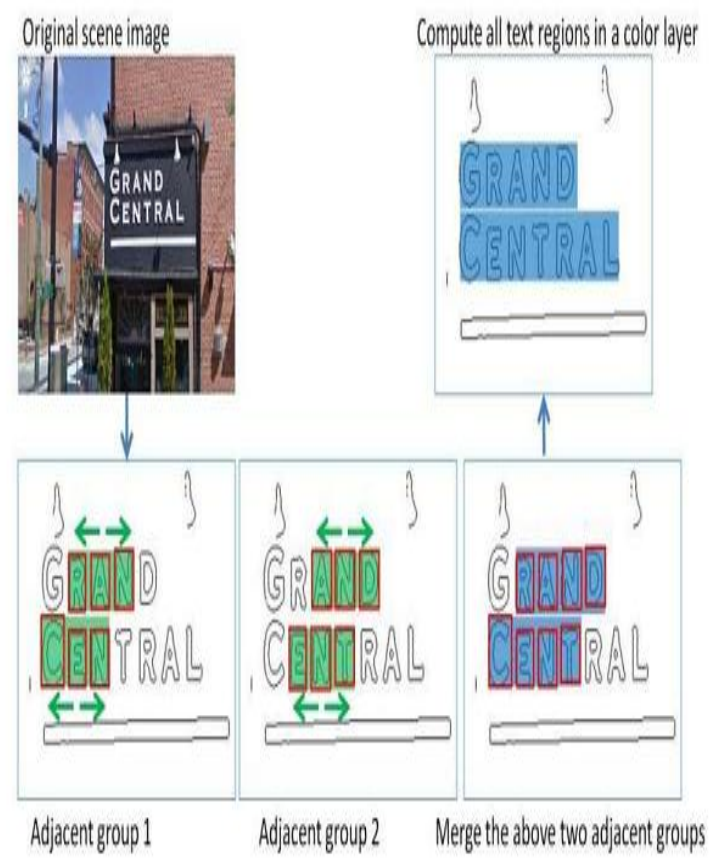

Fig. 2. The adjacent character grouping process. The red box denotes bounding box of a boundary in a color layer. The green regions in the bottom left two figures represent two adjacent groups of consecutive neighboring bounding boxes in similar size and horizontal alignment. The blue regions in the bottom-right figure represent the text string fragments, obtained by merging the overlapping adjacent groups.

\section{Extract Text Strings}

In order to extract text strings in slightly non-horizontal orientations, we search for possible characters of a text string within a reasonable range of horizontal orientation. When estimating horizontal alignment, we do not require all the characters exactly align in horizontal orientation, but allow some differences between neighboring characters that are assigned into the same string. In our system we set this range as $\pm p / 6$ degrees relative to the horizontal line. This range could be set to be larger but it would bring in more false positive strings from background. In addition, our scene text detection algorithm can handle challenging font variations

To be compatible with blind-assistant demo system, some technical details of our scene text detection algorithm are adjusted. At first the input image is down-sampled to improve the efficiency.

\section{SCENE TEXT RECOGNITION}

Character recognition is performed to extract text information. In current work, scene text characters include 10 digits [0-9] and 26 English letters in upper case [A-Z] and lower case $[\mathrm{a}-\mathrm{z}]$. Three public datasets are employed for training character recognizer and

evaluating its performance, and these datasets contain image patches of complete and regular text characters cropped from scene images. We will provide detailed descriptions in Section VI.
As mentioned in Section I, we design two character recognition schemes. In text understanding, character recognition is a multi-class classification problem. We train a character recognizer to classify the 62 classes of characters. In text retrieval, character recognition is a binary classification problem. For each of the 62 character classes, For example, we train a binary classifier for character class ' $\mathrm{A}$ ', then this classifier will predict a patch containing ' $A$ ' as positive, and predict a patch containing other character classes or non-text outliers as negative. The specified character classes are defined as queried characters.

In both schemes, a robust character descriptor is required to extract structure features from character patches. In text retrieval, to better model character structure, we define stroke configuration for each character class based on specific partitions of character boundary and skeleton.

\section{A. Character Descriptor}

A novel character descriptor to model character structure for effective character recognition is proposed here. Fig. 5 depicts the flow chart of our proposed character descriptor. It employs four types of key point detectors, Harris detector (HD) to extract key points from corners and junctions, MSER detector (MD) to extract key points from stroke components, Dense detector (DD) to uniformly extract key points, and Random detector (RD) to extract the preset number of key points in a random pattern.

At each of the extracted key points, the HOG feature is calculated as an observed feature vector $x$ in feature space. Each character patch is normalized into size $128 \times 128$, containing a complete character. In the process of feature quantization, the Bag-of-Words (BOW) Model and Gaussian Mixture Model (GMM) are employed to aggregate the extracted features.

1) Bag-of-Words Model (BOW): The BOW representation is computationally efficient and resistant to intra-class variations. At first, $k$-means clustering is performed on HOG features extracted from training patches to build a vocabulary of visual words. Then feature coding and pooling are performed to map all HOG features from a character patch into a histogram of visual words. We adopt soft-assignment coding and average pooling schemes in the experiments. More other coding/pooling schemes will be tested in our future work.

This number of visual words is experimentally chosen to balance the performance of character recognition and the computation cost. At a character patch, the four detectors are applied to extract their respective key points, and then their corresponding HOG features are mapped into the respective vocabularies, obtaining four frequency histograms of visual words. Each histogram has 256 dimensions. Then we cascade the four histograms into BOW-based feature representation in $256 \times 4=1024$ dimensions.

2) Gaussian Mixture Model (GMM): In our experiments, DD generates a uniform $8 \times 8$ key point array and RD generates 64 key points randomly, but all character 
patches share the same random pattern. Therefore, the key points extracted by RD and DD are always located at the same positions in all character patches, as shown in Fig. 6. To describe the local feature distributions, we build a GMM over all character patches in training set. In our experiments, each GMM contains 8 Gaussian distributions. This parameter is selected from the best results of scene character recognition.

In the process of building GMM, $K$-means clustering ( $K=$ 8 ) is first applied to calculate $K$ centers of the HOG descriptors, where the $s$-th $(1=s=K)$ center is used as initial means of the $s$-th Gaussian in GMM. Then the initial weights and co-variances are calculated from the means. Next, an EM algorithm is used to obtain maximum likelihood estimate of the three parameters, weights, means, and co-variances of all the Gaussian mixture distributions

\section{B. Character Stroke Configuration}

In text retrieval application, the query character class is considered as an object with fixed structure, and we generate its binary classifier according to structure modeling. Character structure consists of multiple oriented strokes, which serve as basic elements of a text character.

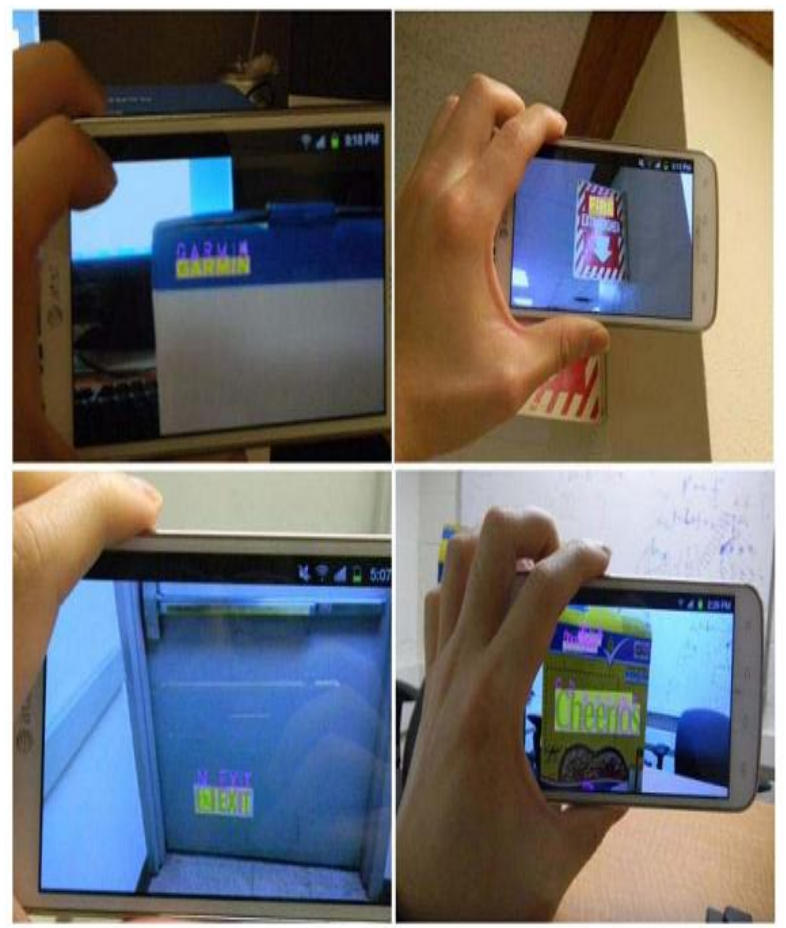

Fig. 3. Our demo system of scene text extraction in Android platform. The text strings "GARMIN", "FIRE", "EXIT", and "Cheerios" are extracted from complex background. Although some characters are incorrectly recognized in current demo, we will introduce lexicon analysis to improve the recognition performance.

From the pixel-level perspective, a stroke of printed text is defined as a region bounded by two parallel boundary segments. In order to locate stroke accurately, stroke is redefined in our algorithm as skeleton points within character sections with consistent width and orientation.
We have developed demo systems of scene text extraction in Android-Mobile platforms. We integrate the functional modules of scene text detection and text recognition. It is able to detect regions of text strings from cluttered background, and recognize characters in the text regions.

In blind-assistant application, the demo system has been used to assist blind or visually impaired people to recognize hand-held object. Another demo system consists of camera, processing device, and Bluetooth earplug. In this system, our proposed method is implemented and deployed into the processing device. Camera is used as input device for capturing natural scene, and Bluetooth earplug is used as output device for broadcasting the recognized text information.

\section{EXPERIMENTAL ANALYSIS}

The main technical contributions of this paper are the two scene character recognition schemes compatible with mobile applications. We perform experiments to evaluate the two schemes over benchmark datasets.

\section{A. Datasets}

To evaluate the proposed character descriptor and the character stroke configuration, we employ three public datasets of scene text characters, in which we conduct scene character recognition. The first one is Chars $74 \mathrm{~K}$ EnglishImg Dataset. It contains all the 62 character classes with the approximately balanced number of samples. The samples in this dataset are divided into two categories, GoodImg and BadImg, according to the recognition difficulty. The second one is Sign Dataset it captures 96 camera-based signs with 1209 scene characters. The third one is ICDAR-2003 Robust Reading Dataset. It contains about 11600 character samples which are cropped from text regions of natural scene images. In Sign Dataset and ICDAR-2003 Dataset, the number of character samples from different categories is unbalanced.

\section{B. Scene Character Recognition for Text Understanding}

Accuracy rate (AR) as evaluation measure, which is defined as the ratio between the number of correctly recognized text characters and the total number of text characters. We further evaluate the two feature representations of our character descriptor independently. BOW-based feature representation obtains 0.53 and GMM-based feature representation 0.47 . This may be due to the fact that BOW-based representations cover key points from all four detectors, while GMM-based representations rely only on DD and RD key points. In DD and $\mathrm{RD}$, it is unavoidable that some key points are not located on characters.

C. Scene Character Recognition for Text Retrieval

The proposed character structure modeling is applied to extract structure features from stroke configuration of the characters to learn a binary classifier for each character class. We evaluate these binary classifiers by queried character classification in the above three datasets. In each character class, two measurements, accuracy rate (AR) and false positive rate (FPR), are calculated to evaluate the performance of queried character classification. 


\section{VII.CONCLUSION}

Our proposed character descriptor is effective to extract representative and discriminative text features for both recognition schemes. To model text character structure for text retrieval scheme, we have designed a novel feature representation, stroke configuration map, based on boundary and skeleton. Quantitative experimental results demonstrate that our proposed method of scene text recognition outperforms most existing methods.

We have also implemented the proposed method to a demo system of scene text extraction on mobile device. In this paper, the accuracy rate of text detection and adding lexicon analysis is done to extend our system to wordlevel recognition in natural videos. To improve the accuracy and practicality of scene text extraction, designing more representative and discriminative features to model text structure will be made.

This can be achieved by collecting a database of specific scene text words as stronger training set, for example, a set of word patches "EXIT" or "SALE" cropped from scene images. In addition, we will combine scene text extraction with other techniques like content-based image retrieval to develop more useful vision based assistant system.

\section{REFERENCES}

[1] R. Beaufort and C. Mancas-Thillou, "A weighted finite-state framework for correcting errors in natural scene OCR," in Proc. 9th Int. Conf. Document Anal. Recognit., Sep. 2007, pp. 889-893.

[2] X. Chen, J. Yang, J. Zhang, and A. Waibel, "Automatic detection and recognition of signs from natural scenes," IEEE Trans. Image Process., vol. 13, no. 1, pp. 87-99, Jan. 2004.

[3] N. Nikolaou and N. Papamarkos, "Color reduction for complex document images," Int. J. Imag. Syst. Technol., vol. 19, no. 1, pp. 14-26, 2009.

[4] A. Coates et al., "Text detection and character recognition in scene images with unsupervised feature learning," in Proc. ICDAR, Sep. 2011, pp. 440-445.

[5] N. Dalal and B. Triggs, "Histograms of oriented gradients for human detection," in Proc. IEEE Conf. Comput. Vis. Pattern Recognit., Jun. 2005, pp. 886-893.

[6] T. de Campos, B. Babu, and M. Varma, "Character recognition in natural images," in Proc. VISAPP, 2009.

[7] B. Epshtein, E. Ofek, and Y. Wexler, "Detecting text in natural scenes with stroke width transform," in Proc. CVPR, Jun. 2010, pp. 2963-2970.

[8] P. F. Felzenszwalb, R. B. Girshick, D. McAllester, and D. Ramanan, "Object detection with discriminatively trained partbased models," IEEE Trans. Pattern Anal. Mach. Intell., vol. 32, no. 9, pp. $1627-1645$, Sep. 2010.

[9] T. Jiang, F. Jurie, and C. Schmid, "Learning shape prior models for object matching," in Proc. CVPR, Jun. 2009, pp. 848-855.

[10] S. Kumar, R. Gupta, N. Khanna, S. Chaudhury, and S. D. Johsi, "Text extraction and document image segmentation using matched wavelets and MRF model," IEEE Trans. Image Process., vol. 16, no. 8, pp. 2117-2128, Aug. 2007.

\section{BIOGRAPHY}

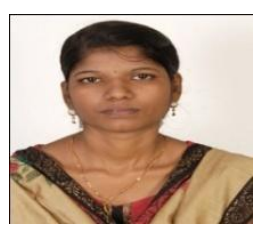

Dhiveha.S received her B.E degree in computer science and engineering in P.R engineering college, Thanjavur in 2013, Tamilnadu, India. Now she is doing her master in engineering in St.Joseph's college of engineering and technology, Thanjavur, Tamilnadu, India. She has attended 2 national conferences, 1 international Journal and 1 international conference. 